\title{
Fabrication of Ge Nanocrystals Doped Silica-on-Silicon Waveguides and Observation of Their Strong Quantum Confinement Effect
}

\author{
Ou, Haiyan; Rottwitt, Karsten
}

Published in:

21th Annual Lasers and Electro Optics Society Meeting

Link to article, DOI:

10.1109/LEOS.2008.4688517

Publication date:

2008

Document Version

Publisher's PDF, also known as Version of record

Link back to DTU Orbit

Citation (APA):

Ou, H., \& Rottwitt, K. (2008). Fabrication of Ge Nanocrystals Doped Silica-on-Silicon Waveguides and Observation of Their Strong Quantum Confinement Effect. In 21th Annual Lasers and Electro Optics Society Meeting (pp. 119-120). IEEE Press. https://doi.org/10.1109/LEOS.2008.4688517

\section{General rights}

Copyright and moral rights for the publications made accessible in the public portal are retained by the authors and/or other copyright owners and it is a condition of accessing publications that users recognise and abide by the legal requirements associated with these rights.

- Users may download and print one copy of any publication from the public portal for the purpose of private study or research.

- You may not further distribute the material or use it for any profit-making activity or commercial gain

- You may freely distribute the URL identifying the publication in the public portal 


\title{
Fabrication of Ge nanocrystals doped silica-on-silicon waveguides and observation of their strong quantum confinement effect
}

\author{
Haiyan Ou, Karsten Rottwitt \\ DTU Fotonik, Department of Photonics Engineering, Technical University of Denmark (DTU), Building 345v, \\ 2800, Kgs. Lyngby, Denmark
}

\begin{abstract}
Standard silica-on-silicon waveguides with a core doped by Ge nanocrystals were fabricated using PECVD and RIE. Transmission of the waveguide was measured, and strong absorption peaks at 1056.8nm, 1406nm and 1263.2nm were observed.
\end{abstract}

\section{Introduction}

During the past decades, germanium (Ge) nanocrystals (nc), also called nanoclusters or quantum dots, have undergone intensive theoretical and experimental research, not only due to their unique electrical and optical properties brought by the quantum confinement effect, but also in light of the potential new devices based on these properties.Among these, Ge nc embedded in a silica matrix material have been mostly investigated for potential applications as light emitters, non-volatile optical memory and enhanced third-order optical nonlinear effects, respectively. One of the major challenges in these efforts has been a small interaction volume. For example; when characterizing $\mathrm{Ge}$ nc with respect to optical nonlinearity high power lasers are needed (typically hundreds of milliwatts). This is due to the small Ge nc film thickness $(\mu \mathrm{m})$.

In this paper, we demonstrate Ge nc doped into the core of a silica waveguide. By coupling light in and out of the waveguide, the interaction length between Ge $\mathrm{nc}$ and light is increased by five orders of magnitude, from submicrometers to centimetres, which not only relaxes the requirement to the power levels of a probe light source, but also eliminates thermal issues. Ge nc doped waveguides also facilitates characterization of nonlinear behaviour by the use of well established optical waveguide theory and experimental technology.

\section{Experiments}

The fabrication of a Ge nc doped waveguide is a combination of processing of standard waveguides and formation of Ge nc. The main steps are plasma enhanced chemical vapour deposition (PECVD) and reactive ion etching. The details of the standard waveguide fabrication and Ge nc formation can be found in [1] and [2], respectively.

Optical characterization was carried out by using a standard waveguide component characterization setup. It consists of a broadband source, a polarizer, a polarization controller (PC), and an optical spectral analyzer (OSA). The Ge nc doped waveguide was placed in a xyz 3D adjustable stage. The light was coupled in and out of the waveguides using standard fibers. An $1.2 \mathrm{~cm}$ long Ge nc doped waveguide was measured and compared against a standard waveguide of the same length using the same setup. All measurements were made at room temperature.

\section{Results and discussions}

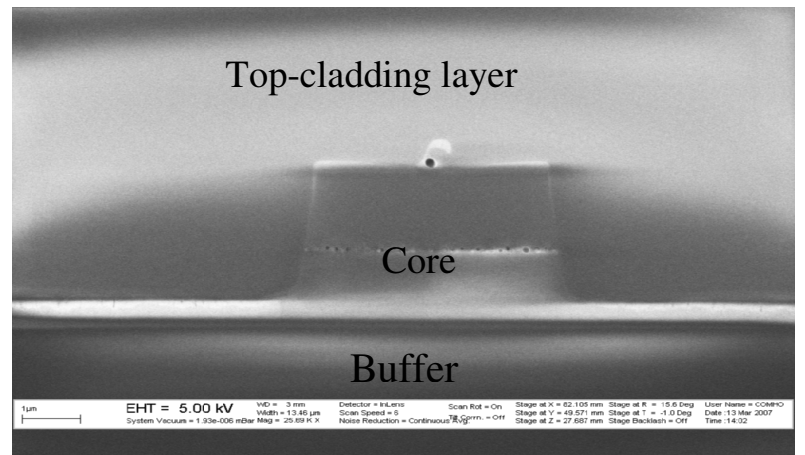

Fig. 1 (a) SEM cross-sectional image of Ge nc doped waveguide. A $3 \times 4$ $\mu \mathrm{m}^{2}$ core is buried in the top-cladding and the buffer. A strip containing Ge $\mathrm{nc}$ is sandwiched close to the middle position of the core.

The cross-section of the Ge nc doped waveguide was investigated with a scanning electron microscope (SEM), see Fig. 1. Fig. 1(a) shows that the core is buried in the buffer and top-cladding layer of the waveguide. A strip of Ge nc doped germanosilicate layer is sandwiched in the middle of the core. Fig. 1(b) is a zoom in of the area containing the 
Ge nc. It is noted that the Ge nc varies in size. The maximum diameter is $140 \mathrm{~nm}$. It is also noted that only a very small fraction of the core is doped by Ge nc. The reason for this is to avoid an extremely high scattering loss of the waveguide due to the Ge nc. In addition, by using relatively low Ge nc concentration, the waveguide is kept single moded at dimensions similar to dimensions of standard silica-on-silicon integrated lightwave circuits. Finally, this design also has flexibility that the fraction of Ge nc in the core may be adjusted simply by adding more strips, as shown in [2].

Measured transmission spectra of a $1.2 \mathrm{~cm}$ long standard waveguide and a $\mathrm{Ge}$ nc doped waveguide of the same length are shown in Fig. 2 (a), together with output spectrum of the broad band light source. The standard waveguide has very low propagation loss, in the order of $0.02 \mathrm{~dB} / \mathrm{cm}$ [1]. Therefore, the total $5 \mathrm{~dB}$ loss is mostly caused by mode mismatch of the standard waveguide to fibers.

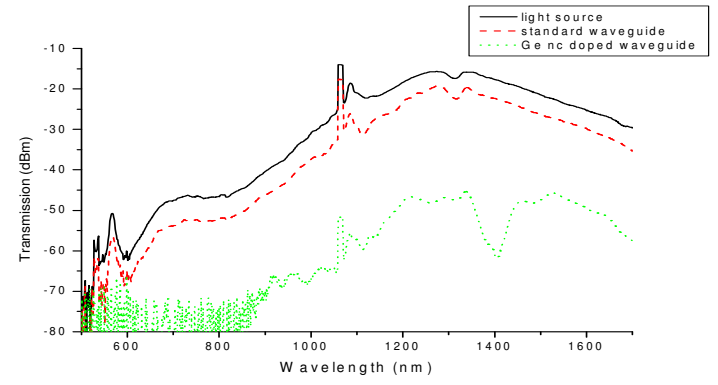

Fig. 2 (a) Spectra of the light source output (solid line), transmission of standard waveguide (dash line) and Ge nc doped waveguide (dot line)

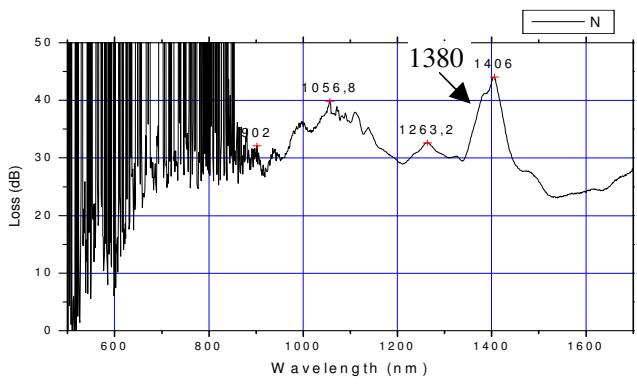

Fig.2 (b) Absorption curve of Ge nc doped waveguide

From the data in Fig. 2(a) the absorption of the Ge nc doped waveguide is obtained by subtracting the transmission spectrum of the Ge nc doped waveguide from that of the standard waveguide, shown in Fig.2(b). The resulting loss represents the pure loss due to the Ge nc, assuming that the coupling losses are the same. Fig.2(b) shows that the loss caused by Ge nc is $22 \mathrm{~dB}$ at $1550 \mathrm{~nm}$, which is $18 \mathrm{~dB} / \mathrm{cm}$. At wavelengths shorter than $900 \mathrm{~nm}$, the waveguide is very lossy. Most important in Fig. 2(b) is the local absorption peaks. Within the wavelength range from 900nm to $1700 \mathrm{~nm}$, there are 3 dominant peaks located at $1056.8 \mathrm{~nm}, 1263.2 \mathrm{~nm}$ and $1406 \mathrm{~nm}$ respectively. The peak at $1406 \mathrm{~nm}$ is very sharp, while the $1056.8 \mathrm{~nm}$ peak is very broad and has several satellite peaks. The peak at $1406 \mathrm{~nm}$ is in agreement with the results obtained by Kuo et al [3], who also observed a strong exciton peak at $1408 \mathrm{~nm}$. In addition to this peak Kuo et al also identified a peak at $1360 \mathrm{~nm}$. They attributed their peaks at $1408 \mathrm{~nm}$ and $1360 \mathrm{~nm}$ to electron-to-heavy-hole and electron-to-light-hole transitions, respectively. It is noted that the results of Kuo et al were obtained from Ge quantum wells deposited on a silicon substrate.

The peak reported by Kuo et al, at $1408 \mathrm{~nm}$ corresponds to our peak at $1406 \mathrm{~nm}$ whereas the peak reported by Kuo et al at $1360 \mathrm{~nm}$ appears as a shoulder at $1380 \mathrm{~nm}$ closely collocated with the strong absorption peak centered at $1408 \mathrm{~nm}$. Thus we attributed our peak at $1408 \mathrm{~nm}$ and the shoulder at $1380 \mathrm{~nm}$ to the same transitions as reported by Kuo et al. Finally, the two clear exciton peaks are observed at room temperature, which may also be explained by substantial confinement of the electron states in the Ge nc.

\section{Conclusion}

Ge nc were successfully embedded in a $140 \mathrm{~nm}$ thick strip, located in the center of a $3 \times 4 \mu \mathrm{m}^{2}$ core of silica-on-silicon waveguides by using standard processing of PECVD and RIE. An absorption spectrum of the Ge nc doped waveguide is obtained using standard fibers coupled to a broadband light source. Even at room temperature, three strong absorption peaks at $1406 \mathrm{~nm}, 1263.2 \mathrm{~nm}$ and $1056.8 \mathrm{~nm}$ were observed. We assigned the strong peak at $1406 \mathrm{~nm}$ and the shoulder at $1380 \mathrm{~nm}$ to the electron-to-heavy-hole and electron-to-light-hole transitions respectively, due to the strong quantum confinement in the Ge nc. By doping the core of a waveguide with Ge nc a method has been demonstrated where the interaction length $(1.2 \mathrm{~cm})$ between $\mathrm{Ge} \mathrm{nc}$ and light is increased dramatically, compared against a small film thickness (140nm) which is usually used to characterize Ge nc.

*The Danish Technical Research Council is thanked for financial support.

\section{References}

1. H. Ou, Electron. Lett. 39, 212-213 (2003)

2. H. Ou, T.P. Rørdam, K. Rottwitt, et al, Appl. Phys. B 91, 177-181 (2008)

3. Y.-H. Kuo, Y. K. Lee, Y. Ge, et al, Nature 437, 1334-1336 (2005). 